\title{
PRÁCTICAS DE PREPARACIÓN Y CONSERVACIÓN DE FRIJOLES EN FAMILIAS COSTARRICENSES ${ }^{1}$
}

\author{
Shirley Rodríguez-González², Xinia Elena Fernández-Rojas ${ }^{2}$
}

\begin{abstract}
RESUMEN
Prácticas de preparación y conservación de frijoles en familias costarricenses. El objetivo de este trabajo fue identificar las prácticas de preparación y de conservación de frijoles en dos poblaciones, una rural (Jesús de Atenas) y una urbana (La Unión de Tres Ríos) de Costa Rica. El estudio fue desarrollado en el 2005, en una muestra de 475 familias que acostumbraban preparar y consumir frijoles (223 de la zona rural y 252 de la zona urbana). En cada una de estas viviendas se entrevistó a la persona encargada de cocinar la mayor parte del tiempo, por lo que los resultados están basados en la información suministrada por esta. El remojo de los frijoles previo a la cocción fue más utilizado en la zona rural $\left(x^{2}=14,435, p<0,001\right)$ y los métodos empleados en esta zona difirieron significativamente de los que se utilizaban en la zona urbana $\left(x^{2}=28,535, p<0,001\right)$. En la zona rural los más utilizados fueron la cocción a presión y la convencional, mientras que en la zona urbana, la cocción a presión. Los métodos de conservación aplicados a los frijoles cocidos no difirieron por zona, siendo el más utilizado la refrigeración $\left(\mathrm{x}^{2}=3,722, \mathrm{p}=0,293\right)$.
\end{abstract}

Palabras claves: preservación de alimentos, zona rural,

\begin{abstract}
Preparation and conservation practices of beans in Costa Rican families. The objective of this work was to identify the culinary and preservation practices for beans in a population rural (Jesus of Atenas) and an urban (La Union of Tres Rios) of Costa Rica. The study was developed in 2005, in a sample 475 families that prepare and consume beans (223 rural and 252 urban). In each of these homes, the person responsible for food preparation was interviewed and it is the key informant. The soaking beans before cooking was used mostly in the rural area $\left(x^{2}=14.435\right.$, $\mathrm{p}<0,001)$ and cooking methods employed in this area differ significantly from those used in the urban area $\left(x^{2}=28.535\right.$, $\mathrm{p}<0,001)$. In the rural area the most used cooking methods were conventional cooking and pressure cooking, but in the urban area the most common method was pressure cooking. The beans preservation methods applied to cooked beans do not different by area, the most common method found was refrigeration $\left(\mathrm{x}^{2}=3.722, \mathrm{p}=0.293\right)$.
\end{abstract}

Keywords: food preservation, rural, urban, culinary practices. zona urbana.

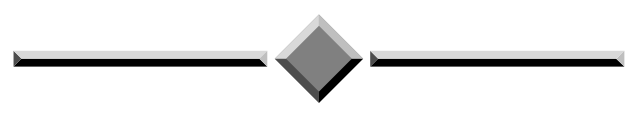

\footnotetext{
1 Recibido: 13 de marzo, 2014. Aceptado: 23 de setiembre, 2014. Parte de tesis para optar por el grado de Magíster Scientiae en Nutrición Humana de la primer autora, Universidad de Costa Rica, dentro del proyecto "Estudio de las características físico-químicas y nutricionales de frijoles (Phaseolus vulgaris)".

2 Universidad de Costa Rica, Escuela de Nutrición. Dirección postal: 2060, Ciudad Universitaria Rodrigo Facio, San José, Costa Rica. shirley. rodriguez@ucr.ac.cr, xinia.fernandezrojas@ucr.ac.cr
} 


\section{INTRODUCCIÓN}

Los frijoles (Phaseolus vulgaris) son una de las fuentes más importantes de energía y nutrientes a nivel mundial, especialmente en países en vías de desarrollo. Los frijoles son una buena fuente de proteína (Bressani y Elías, 1988; Blanco y Bressani, 1991; Messina, 1999), de vitaminas (tiamina y ácido fólico) y de minerales (magnesio, zinc, hierro y fósforo) (Messina, 1999; Menchú y Méndez, 2006; Ramírez et al., 2011). Además, una excelente fuente de carbohidratos y de fibra tanto soluble como insoluble (Menchú y Méndez, 2006; USDA, 2014).

Los frijoles también contienen unas sustancias que limitan tanto la calidad nutricional como la absorción y la disponibilidad de ciertos nutrientes. Estas sustancias han sido llamadas como factores antinutricionales. Los factores tradicionalmente considerados como antinutricionales presentes en los frijoles son: inhibidores de tripsina, las hemaglutininas, los fitatos, los compuestos fenólicos y los oligosacáridos (Elías, s.f.; Bressani et al., 1988; Messina, 1999; Carbonaro et al., 2000; López et al., 2002).

Sin embargo, según Andlauer et al. (1998), Guzmán-Maldonado et al. (2002) y Ulloa et al. (2011) los componentes que históricamente fueron considerados como factores antinutricionales de las leguminosas, en los últimos años se ha demostrado que están relacionados con la prevención o el tratamiento de ciertas enfermedades, sobre todo a dosis bajas como es posible encontrarlas en las formas habituales de preparación para su consumo, como por ejemplo por medio de cocción.

Las sustanciascomo fitatos, flavonoides,cumarinas, triterpenos, lignanos, fenoles y oligosacáridos (Andlauer et al., 1998; Guzmán-Maldonado, 2002; Ramírez et al., 2011) han sido identificados como elementos protectores contra algunos de los factores que estimulan el crecimiento de los tumores y el envejecimiento de los tejidos (Singh y Fraser, 1998; Kolonel et al., 2000; Slavin, 2000; Rodríguez y Fernández-Rojas, 2003) y además, ayudan a reducir el riesgo de padecer otras enfermedades crónicodegenerativas (Bazzano et al., 2001; Osorio et al., 2003; Rodríguez y Fernández-Rojas, 2003; Serrano y Goñi, 2004; Ryan et al., 2007).

Es por eso que las leguminosas, han sido catalogadas como un "alimento funcional"; es decir, un alimento que además de nutrientes, aporta otras sustancias que tienen un efecto benéfico sobre la salud (Guzmán-Maldonado et al., 2002; Ulloa et al., 2011).

Según Rodríguez y Fernández-Rojas (2003) este alimento, por su composición nutricional, ha jugado un papel fundamental en el mantenimiento y la sostenibilidad del estado nutricional de la población costarricense.

Los frijoles requieren de la aplicación de técnicas culinarias para su preparación y conservación; al respecto, la literatura informa de importantes cambios en el contenido de nutrientes hidrosolubles y termolábiles, asociado con la técnica aplicada (FAO, 1982; FAO, 1990; Payne et al., 1994; Jiménez y Leal, 1996; Chau y Cheung, 1997; Tacsan, 2001; Reynoso et al., 2007). En Costa Rica, hay poca información sobre las prácticas culinarias. El único trabajo que identifica prácticas culinarias es el realizado por Campos et al. (1999), el cual se circunscribió a una población urbana de clase media.

Considerando que no hay información comparativa entre las prácticas realizadas para preparar y conservar los frijoles entre una población urbana y una población rural, este estudio pretende aportar en este conocimiento. La comparación de las zonas se justifica en el sentido de que los contextos en los que las personas están involucradas pueden influir en sus prácticas de manejo de los frijoles, pues los modos de vida de la zona rural y la zona urbana son diferentes. Las personas en la zonas urbanas tienen menos tiempo disponible tanto para preparar los alimentos como para su consumo, pero también, disponen de mayor disponibilidad de alimentos procesados prontos para su consumo (García, 1997, 2003), aspectos que son contrastantes con la zona rural donde todavía la persona que prepara los alimentos dispone de más tiempo para comprar, preparar y consumir alimento, y se procura una alimentación más tradicional a partir de alimentos frescos, los cuales son más disponibles y a un menor costo que en la zona urbana.

Es necesario realizar estudios que permitan identificar las prácticas relacionadas con el manejo de este alimento en el hogar, con el fin de establecer recomendaciones que permitan preservar el mayor contenido de nutrientes y aumentar el consumo de frijoles. Una mejor preparación y un mayor consumo de este alimento mejorará el aporte de nutrientes a la dieta de los costarricenses, lo que se traducirá en un beneficio para la salud de la población. A partir de este 
interés, el objetivo de este estudio fue identificar las prácticas de preparación y de conservación de frijoles en familias de dos comunidades, una urbana y una rural de Costa Rica.

\section{MATERIALES Y MÉTODOS}

Este estudio fue de tipo transversal y descriptivo con enfoque cuantitativo que buscaba determinar las prácticas de preparación y conservación de frijoles en dichas zonas y que podrían afectar la calidad nutricional del grano.

El estudio se desarrolló en el 2005 en una población rural y una urbana de Costa Rica. La población estuvo constituida por las familias de las comunidades de Jesús de Atenas, población rural y La Unión de Tres Ríos, población urbana.

La muestra fue seleccionada a partir de un muestreo estratificado por conglomerados según condición geográfica. Para definir la muestra se tomaron en cuenta los siguientes criterios: varianza y promedio de la variable de consumo de frijoles de la Encuesta Nacional de Consumo de Alimentos (Ministerio de Salud, 2001; confianza de 95\% y error del 10\%). Se obtuvo un total 475 familias, 223 familias en la zona rural y 252 familias en la zona urbana. En dichas familias fue entrevistada la persona que era la responsable de preparar y de conservar los alimentos en el hogar y que voluntariamente aceptó participar en el estudio. Se partió del supuesto de que la persona que prepara los alimentos en el hogar también prepara los frijoles para el consumo de la familia.

Entre las limitaciones del estudio pueden citarse: no se midieron los conocimientos y las percepciones con respecto a los frijoles de la persona encargada de preparar los alimentos, ni las características de las viviendas que podrían influir en las prácticas de preparación y conservación.

Las variables estudiadas fueron:

1. Localización geográfica.

2. Característica de la persona encargada de preparar los alimentos en el hogar.

3. Prácticas de preparación:

a. Remojo: aplicación o no y características de este.

b. Cocción: método de cocción y características de este.

4. Prácticas de conservación: tipo y características.
Estas variables fueron definidas a partir del referencial teórico y de estudios precedentes sobre prácticas asociadas a la preparación, consumo y almacenamiento de frijoles en Costa Rica.

Para recolectar los datos se elaboró un cuestionario semiestructurado que fue validado en una población similar y que posteriormente fue aplicado a la muestra. Considerando las variables del estudio, este cuestionario permitió obtener la información referente a las características generales de la familia, las de la persona encargada de preparar los alimentos y las prácticas de preparación y conservación de frijoles en las familias.

Todas las variables en estudio fueron analizadas a partir de estadística descriptiva y a partir de estadística inferencial con el fin de establecer las diferencias entre las zonas analizadas. La prueba estadística utilizada fue Chi-cuadrado, con un nivel de confianza del $95 \%$. Los datos fueron analizados con el programa estadístico SPSS versión 12.

\section{RESULTADOS Y DISCUSIÓN}

\section{Caracterización de la muestra}

Las familias estudiadas tenían un promedio de cuatro miembros, donde el $71 \%$ tenían como jefe de hogar al padre, $19 \%$ a la madre, $4 \%$ a un hijo y un $6 \%$ a otro miembro de la familia.

La mayoría de las personas encargadas de preparar los alimentos en el hogar son mujeres con edades que van entre 38 y 59 años, con un promedio de edad de 48 años $\pm 15,5$; este promedio de edad difirió por zona, en la zona urbana fue de 50,0 $\pm 14,7$ y en la zona rural de $46,8 \pm 16,3$.

El nivel de escolaridad de la persona encargada de preparar los alimentos difirió por zona, en la zona rural la mayoría de las personas que están a cargo de preparar los alimentos tuvieron un nivel de escolaridad inferior a primaria completa, mientras que en la zona urbana la mayoría presenta un nivel educativo superior a secundaria incompleta.

Las personas encargadas de preparar los alimentos fueron en su mayoría amas de casa en ambas zonas estudiadas. Sin embargo, en la zona urbana un $36 \%$ de las personas encargadas de la preparación de alimentos también poseían un trabajo remunerado, mientras que en la zona rural solamente un $24 \%$ de ellas. 
Los datos revelaron que un $92 \%$ de las personas que preparaban los alimentos en ambas zonas también eran las que decidían lo que se prepara en el hogar.

\section{Prácticas de preparación de frijoles}

\section{Prácticas de remojo}

El procedimiento de remojo antes de cocinar los frijoles fue realizado en $170(36 \%)$ hogares de la muestra estudiada, siendo mayor el número de familias de la zona urbana (23\%) en comparación con las de la zona rural $(13 \%)\left(x^{2}=14.435, p<0,001\right)$. En ambos casos el porcentaje es bajo, dadas las ventajas de esta práctica y su conveniencia para reducir el gasto de energía.

Las razones que brindaron las familias que remojaban los frijoles para hacerlo fueron: hidratar y suavizar el grano (64\%), eliminar sustancias que producen daño (coliquientas o tóxicas) (21\%), economizar energía (8\%), eliminar suciedad $(1 \%)$ y un $6 \%$ no manifestaron la razón.

Por su parte, las razones que dieron las familias para no realizar el remojo de los frijoles previo a su cocción fueron: la suavidad del grano que consumen (35\%), la técnica de cocción que aplican no lo requiere (20\%) y porque no es una costumbre adquirida (31\%). En la zona urbana, una razón importante para no llevar a cabo esta práctica, a pesar de que son las que más remojan, fue el poco tiempo del que se dispone para realizarla (9\%).

Este porcentaje de familias que remojaban los frijoles fue concordante con otros estudios, pues según Campos et al. (1999) remojar los frijoles antes de cocinarlos es una práctica realizada por muy pocas mujeres en una población urbana.

El hecho de que en pocas familias se aplica esta práctica puede deberse a dos razones: las personas encargadas de preparar los alimentos tienen menos información sobre los beneficios de realizarla o que conocen la información pero esta no es importante para ellas, ya que las razones que mencionaron para no aplicar el remojo están relacionadas con la técnica de cocción y con las características del grano, pero ninguna estuvo relacionada con la salud. Sin embargo, esta práctica debe recomendarse, debido a que este procedimiento disminuye el tiempo de cocción, reduce los problemas digestivos asociados a su consumo y favorece la conservación de los nutrientes del grano (FAO, 1990; Ramírez-Cárdenas y Costa, 2008; Fernández et al., 2010).

El hecho de que el remojar los frijoles antes de la cocción se realizara en menor medida en la zona rural pudo deberse a que las personas de esta zona tienen menos información sobre los beneficios de realizar dicho proceso o porque este no forma parte de la tradición culinaria. A pesar de que, para ambas zonas estudiadas, se dieron las mismas razones para llevar a cabo la práctica, en la zona urbana más familias dieron como razón eliminar sustancias dañinas.

Según Murillo y Rodríguez (2004), una educación adecuada permite la incorporación de dicha práctica en la preparación de los frijoles, lo cual puede favorecer el consumo de este grano, pues una de las razones de no consumo encontradas por Campos et al. (1999) fueron los problemas de digestibilidad asociados al mismo.

El agua que se utilizaba para remojar los frijoles por lo general era agua a temperatura ambiente, únicamente en siete familias de ambas zonas (dos de la zona rural y cinco de la zona urbana) utilizaban agua caliente para realizar dicho procedimiento; esta práctica se considera inadecuada, ya que el uso de agua caliente produce la inhibición de enzimas que intervienen en la degradación de los polifenoles y la hidrólisis de almidones, y además puede afectar la calidad nutricional por exposición más prolongada al calor (Kon, 1979, Hoppner y Lamp, 1993; Lean y Fox, 2006). De acuerdo con lo anterior, el remojo por largo tiempo y con agua a temperatura ambiente es lo más recomendable para las personas que presentan intolerancias.

El agua resultante del proceso de remojo se utilizaba por algunas de las familias como medio de cocción (12 familias de la zona rural (5\%) y 46 familias de la zona urbana (18\%) y otras preferían desecharla para adicionar agua nueva al proceso de cocción (48 familias de la zona rural (21\%) y 64 familias de la zona urbana (24\%).

La principal razón que ofrecieron las personas entrevistadas para eliminar el agua utilizada para remojar los frijoles fue que esta se utiliza para extraer sustancias dañinas para la salud (productos químicos y sustancias coliquientas) (43\%), para lavar los granos (38\%), porque es una costumbre (6\%) o porque les causa desagrado (13\%). Esta práctica puede reducir la calidad nutricional de los frijoles, ya que en el agua de remojo hay sustancias nutritivas en solución que se pierden al desecharla (FAO, 1990, Prodanov y Sierra, 2004), 
pérdida que aún no se ha cuantificado, por lo que esta práctica solo es recomendable para aquellas personas que aun remojando los frijoles, continúan presentando problemas digestivos al consumirlos (Rodríguez y Fernández-Rojas, 2003). Se debe considerar que las personas no tienen la información suficiente para realizar un adecuado uso del agua de remojo.

El tiempo de remojo aplicado en ambas zonas era similar, en la zona rural el tiempo de remojo promedio era de 5,5 horas y en la zona urbana de 5,0 horas. Sin embargo, el tiempo recomendado de remojo ( 8 a 12 horas) era aplicado únicamente por 60 familias (22\%) (14 de la zona rural (6\%) y 46 de la zona urbana (18\%)) y ninguna familia aplicaba el método de remojo rápido tal y como lo indica la literatura.

De las familias que acostumbran remojar los frijoles únicamente catorce adicionaban alguna sustancia durante el remojo; en la zona rural, únicamente dos de ellas, y lo que adicionaban era sal, con el objetivo de suavizar el grano. Por su parte, en la zona rural cuatro adicionaban sal, cuatro bicarbonato, dos condimentos naturales y dos condimentos artificiales; con el objetivo de suavizar y mejorar el sabor de los frijoles cocidos.

Esta práctica fue coincidente con lo encontrado en la literatura, donde el tiempo de cocción y la concentración de taninos se reduce significativamente al adicionar soluciones salinas o bicarbonato (Deshpande y Cheryan, 1983; León et al., 1992; Guzmán et al., 1996; Jiménez y Leal, 1996; Tacsan, 2001; Ramírez-Cárdenas y Costa, 2008). Pese a que estudios demuestran que la adición de bicarbonato de sodio es efectiva para provocar el ablandamiento de los frijoles con una reducción significativa en el tiempo de cocción, es importante rescatar que el bicarbonato de sodio aumenta la destrucción de la tiamina presente en los frijoles durante el proceso de cocción (Lean y Fox, 2006, 1999; Badui, 2006), por lo que esta práctica no es recomendable.

De las 168 familias que remojaban los frijoles previo a su cocción, únicamente 60 (36\%) aplicaban el tiempo de remojo recomendado, 14 de ellas en la zona rural (8\%) y 46 en la zona urbana $(27 \%)$; evidenciando diferencias estadísticas entre zonas $\left(x^{2}=5,809, p=0,016\right)$.

Como se ha discutido, el remojo es una práctica recomendada por expertos. Son dos los motivos básicos para proponer y realizar este procedimiento previo a su consumo y son: la eliminación de los factores antinutricionales presentes en los frijoles crudos y la disminución en el tiempo de cocción (Bressani et al., 1988; Ramírez-Cárdenas y Costa, 2008).

\section{Prácticas de preparación y cocción}

La frecuencia de cocción de frijoles por mes era de 7,2 $\pm 3,3$ veces en la zona rural y de $8,4 \pm 4,8$ veces en la zona urbana, y la cantidad de frijoles cocinada en promedio por ocasión era de $637 \mathrm{~g}$ en la zona rural, mientras que en la zona urbana era de $710 \mathrm{~g}$.

En la zona rural, los métodos más utilizados eran la cocción a presión y la cocción convencional, mientras que en la zona urbana la cocción a presión era la más usada (Cuadro 1).

Según los datos obtenidos hay evidencia estadística para afirmar que el método de cocción empleado para preparar los frijoles difirió por zona $\left(\mathrm{x}^{2}=28,535, \mathrm{p}<0,001\right)$.

Las razones que brindaron las personas encargadas de preparar los alimentos para el uso de la cocción a presión fueron: es un método muy rápido y práctico $(84 \%)$, económico $(7 \%)$, el grano del frijol queda bastante suave (7\%) y porque es la única opción disponible (2\%). Las personas que acostumbraban a cocinar los frijoles en un cocimiento lento manifestaron que es un método de cocción muy práctico que no requiere un control minucioso, el grano queda suave, con gran sabor y el caldo espeso (83\%), ayuda a economizar electricidad $(8 \%)$, les da temor el uso de la olla de presión (5\%) o es la única opción disponible (4\%).

Por su parte, las personas que utilizaban la cocción convencional indicaron que la emplean porque era la opción disponible (42\%), porque el método permite el control de la suavidad del grano (42\%), por temor al uso de la olla de presión (9\%) y por costumbre (7\%).

La mayoría de las familias utilizan la olla de presión para cocinar los frijoles, lo cual es adecuado, ya que mejora su digestibilidad y evita la pérdida excesiva de nutrientes, debido a la rápida cocción (Lean y Fox, 2006).

En la zona rural, un alto porcentaje de las familias utilizaban la cocción convencional para cocinar los frijoles y en menor porcentaje la cocción lenta, mientras que en la zona urbana utilizaban de igual forma el cocimiento lento y la cocción convencional. 
Cuadro 1. Distribución de las familias según método de cocción aplicado para cocinar los frijoles y condición geográfica. Jesús de Atenas y La Unión de Tres Ríos, Costa Rica. 2005.

\begin{tabular}{lcccc}
\hline Método de cocción & \multicolumn{2}{c}{ Rural } & \multicolumn{2}{c}{ Urbano } \\
\cline { 2 - 5 } & Absoluto (n) & Relativo (\%) & Absoluto (n) & Relativo (\%) \\
\hline Cocción a presión & 98 & 44 & 155 & 61 \\
Cocimiento lento & 36 & 16 & 52 & 21 \\
Cocción convencional & 89 & 40 & 45 & 18 \\
Total & 223 & 100 & 252 & 100 \\
\hline
\end{tabular}

El uso de estos métodos no es tan recomendado, ya que el tiempo de cocción prolongado reduce la biodisponibilidad de la lisina y de los aminoácidos azufrados (Bressani, 1989); además provoca la pérdida de algunas vitaminas, destacándose la pérdida de ácido fólico que ocurre progresivamente conforme aumenta el tiempo de cocción (Hoppner y Lamp, 1993; Dang et al., 2000; Badui, 2006).

Campos et al. (1999) en una población urbana de clase media, también encontró el mismo grado de utilización de los métodos de cocción a lo encontrado para la misma zona en este estudio, primero la cocción a presión, después la cocción lenta y por último la cocción convencional. El uso de la olla de cocimiento lento es bastante práctico para las familias, ya que es un método que no requiere un control estricto y que favorece un producto con características agradables a los miembros de la familia (grano suave, gran sabor y caldo espeso).

Durante el proceso de cocción la adición de sal a los frijoles, en ambas zonas, se realizaba en dos momentos: cuando se iban a poner a cocinar los frijoles ( $53 \%$ en la zona rural y $66 \%$ en la zona urbana) y cuando los frijoles estuvieron suaves (47\% en la zona rural y $34 \%$ en la zona urbana).

La adición de sal en el proceso de cocción estuvo asociado al método de cocción aplicado tanto en la zona urbana como en la zona rural $\left(x^{2}=28,535\right.$, $\mathrm{p}<0,001)$, las personas que utilizaban la cocción a presión para cocinar los frijoles adicionaban la sal cuando los iban a poner a cocinar, mientras que las personas que utilizaban el método de cocción convencional la adicionaban cuando el grano ya estaba suave (Figura 1).

Las personas que utilizaban el método de cocción a presión indicaron que se adicionó la sal cuando se iban a poner a cocinar los frijoles porque el método de cocción no permite abrir la olla hasta que estén cocidos, por esta razón es más práctico adicionarla al inicio de la cocción. Sin embargo, algunas personas mencionaron que a pesar de que utilizan ese método adicionaban la sal hasta que estuvieron suaves los frijoles, porque si se agrega antes los frijoles no se suavizan. La adición de la sal en métodos como la cocción lenta y la cocción en olla convencional, se puede realizar en cualquier momento del proceso, ya que se puede levantar la tapa de la olla y controlar el proceso sin ningún peligro. Esta práctica también fue encontrada en la población urbana estudiada por Campos et al. (1999).

En el caso de las familias que cocinaban los frijoles en olla convencional, una razón muy importante para adicionar la sal cuando ya el grano estuviera suave era la creencia que tienen de que si se agrega la sal antes de que estén suaves los frijoles quedan duros; sin embargo, también manifestaron como razones, el delicioso sabor del caldo y del grano, pues este se suaviza y permanece entero. Con respecto a las personas que utilizaban la olla de cocimiento lento, las razones que expresaron para adicionar la sal en el momento referido fue porque era más práctico y porque el grano queda con mejor sabor.

Agregar la sal al final del proceso convencional de cocción es lo adecuado, debido a que si se agrega antes el tiempo de cocción se retarda y se requiere más cantidad de sal para dar la sazón adecuada (Dumani y Rodríguez, 2000; Rodríguez y Fernández-Rojas, 2003), además puede reducir la calidad proteínica (Bressani, 1989).

Con respecto a los productos utilizados para cocinar los frijoles, en la zona rural $95 \%$ de las familias utilizaban únicamente condimentos naturales, 


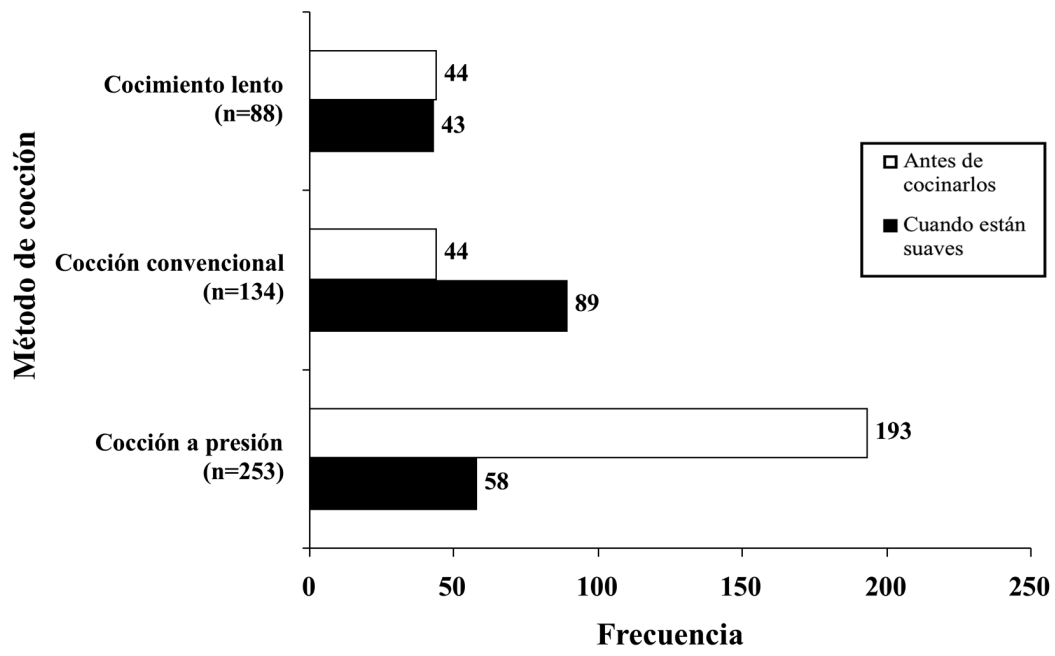

Figura 1. Distribución de las familias según el momento de adición de sal en el proceso de cocción y método aplicado. Jesús de Atenas y La Unión de Tres Ríos, Costa Rica. 2005.

$3 \%$ adicionaban condimentos naturales y artificiales y un $2 \%$ no adicionaban nada. Ninguna de las familias utilizaba grasa en la cocción.

Por su parte, en la zona urbana $88 \%$ familias agregaban únicamente condimentos naturales, $1 \%$ únicamente condimentos artificiales, $8 \%$ condimentos naturales y artificiales, $1 \%$ condimentos naturales y grasa, $2 \%$ no adicionaban nada durante el proceso de cocción. El uso reportado de condimentos artificiales fue bajo, lo cual pudo deberse a que exista un pensamiento de que son conceptualizados como dañinos, no se reportaran o a que son agregados en procesos posteriores de uso del alimento.

En la zona urbana se encontró una mayor variabilidad del uso de los frijoles en la preparación de platillos, ya que el número promedio de preparaciones en las que utilizaban el frijol rojo es de 1,7 y de 1,9 para el frijol negro, mientras que en la zona rural es de 1,1 para el frijol rojo y 0,8 para el frijol negro.

En ambas zonas, los frijoles rojos se incluían en las siguientes preparaciones: gallo pinto, empanadas, emparedados, empanadas o pastel de plátano maduro y frijoles con cerdo. Cabe resaltar que hay preparaciones que se realizaban en una zona y no en la otra, como es el caso de los tamales con frijoles y gallos con torta de huevo, mencionados en la zona rural; mientras que en la zona urbana incluyeron los frijoles como parte de preparaciones rápidas como son los burritos y las chalupas. Por su parte, los frijoles negros eran utilizados en las mismas preparaciones mencionadas para los frijoles rojos, pero además se utilizaban para preparar la tradicional sopa negra.

Debido al limitado uso del frijol en preparaciones, promover la incorporación de este alimento en diferentes platillos puede ser una estrategia adecuada para aumentar el consumo de este grano y dar mayor variedad a la dieta de las familias.

Otra práctica utilizada era la de realizar una preparación previa al consumo de frijoles cocidos, comúnmente llamada "arreglar los frijoles", la cual se define como "acción de condimentar los frijoles en una segunda cocción corta, con condimentos naturales como cebolla, ajo, chile dulce, culantro castilla, pimienta o comino y con condimentos artificiales como consomé y salsa inglesa". En la zona rural 131 familias $(52 \%)$ acostumbraban a realizar dicha práctica y en la zona urbana 166 familias (66\%). En ambas zonas, la mayoría utilizaba condimentos naturales, artificiales y grasa en este tipo de preparación. Resultados similares fueron encontrados en una comunidad urbana en el 
estudio de Campos et al. (1999) donde se utilizaba grasa, condimentos artificiales y condimentos naturales para "arreglar los frijoles".

El uso de condimentos naturales tanto para preparar los frijoles como para "arreglarlos" es positivo, porque esto ayuda a aumentar el valor nutritivo de los frijoles, por el contrario, el uso de grasa y de condimentos artificiales, además de aumentar el valor calórico y el aporte de sodio, puede provocar problemas digestivos y las personas pueden asociar estos al consumo de frijoles y no a la práctica de preparación.

Al analizar las prácticas relacionadas con los procesos de cocción, estas fueron agrupadas como recomendadas o no recomendadas en relación con las que permitieran una mayor conservación de nutrientes. De las 475 familias estudiadas únicamente un 11\% realizaban una cocción recomendada, la cual se refiere a que utilizaban olla de presión para cocinar los frijoles, un tiempo de cocción menor a 90 minutos y agregar la sal cuando los frijoles estaban suaves. Es importante destacar que hubo un $14 \%$ de las familias que no realizaban ninguna de las prácticas recomendadas ni de remojo, ni de cocción.

Muy pocas familias realizaban las prácticas de cocción recomendadas, por lo que es importante educar a la población en aquellas prácticas que pueden conservar en mayor cantidad los nutrientes que ofrecen estos granos.

\section{Prácticas de conservación de frijoles cocidos}

Los métodos de conservación encontradas en ambas comunidades son tres: el hervido diario, la refrigeración y la congelación (Cuadro 2).
La utilización de estos tres métodos en ambas zonas tuvo el mismo comportamiento; el método más utilizado fue la refrigeración, seguido por el hervido frecuente y por último, con un menor uso la congelación.

Cabe destacar que en tres familias de la zona urbana no realizaban la práctica de conservación, ya que cocinaban los frijoles que iban a utilizar en el día.

Estos resultados son concordantes con los reportados por Campos et al. (1999), donde la práctica más utilizada para conservar los frijoles cocidos en la población urbana estudiada era la refrigeración, aunque también se mencionan como métodos utilizados el hervido diario y el congelado.

Los resultados obtenidos evidencian que no hubo diferencias estadísticas en el uso de los diversos métodos de conservación por zona $\left(x^{2}=3,722\right.$, $\mathrm{p}=0$,293), siendo mayor el uso de la refrigeración.

De las personas entrevistadas que manifestaron utilizar la refrigeración como el método de conservación dieron diversas razones, entre ellas, porque mantenía en buenas condiciones la cantidad de frijoles que acostumbran cocinar $(60 \%)$, porque tienen una percepción negativa de otro método de conservación (33\%) y porque es una costumbre (7\%). Por su parte, las personas que acostumbraban utilizar el método de conservación por hervido manifestaron las siguientes razones: les desagrada refrigerar $\mathrm{o}$ congelar los frijoles (51\%), este método les asegura que los frijoles no se descompongan (28\%), y es una costumbre adquirida (21\%). Los que utilizaban la congelación indicaron que les aseguraba mantenerlos por el tiempo requerido (74\%), que ayuda a preservar el valor nutritivo (4\%) y porque tienen una percepción negativa de la refrigeración o el hervido (22\%).

Cuadro 2. Distribución de las familias según método de conservación aplicado a frijoles cocidos y condición geográfica. Jesús de Atenas y La Unión de Tres Ríos, Costa Rica. 2005.

\begin{tabular}{lcccccc}
\hline \multirow{2}{*}{$\begin{array}{c}\text { Método de } \\
\text { conservación }\end{array}$} & \multicolumn{2}{c}{ Rural } & \multicolumn{2}{c}{ Urbano } & \multicolumn{2}{c}{ Total } \\
\cline { 2 - 7 } & Absoluto (n) & Relativo (\%) & Absoluto (n) & Relativo (\%) & Absoluto (n) & Relativo (\%) \\
\hline Refrigeración & 143 & 64 & 151 & 60 & 294 & 62 \\
Hervido diario & 56 & 25 & 73 & 29 & 129 & 27 \\
Congelación & 24 & 11 & 25 & 10 & 49 & 10 \\
No conserva & & & 3 & 1 & 3 & 1 \\
Total & 223 & 100 & 252 & 100 & 475 & 100 \\
\hline
\end{tabular}


Los resultados mostraron que existe relación entre el método utilizado para la conservación y la duración de los frijoles. En las familias que utilizaban el hervido frecuente, la duración en promedio era de cinco días para la zona rural y cuatro para la zona urbana. Por su parte, en las familias que utilizaban la congelación como método de conservación, la duración de los frijoles era de seis días en la zona rural y ocho días en la zona urbana. Mientras que las familias que empleaban el método de conservación en refrigeración, la duración en promedio fue de 5,5 días.

Las familias que utilizaban la refrigeración como método para conservar los frijoles acostumbraban recalentar, en la mayoría de los casos $(86 \%)$, la cantidad de frijoles que iban a utilizar por tiempo de comida y no se encontró diferencias estadísticas por zona.

La mayoría de las familias de la zona urbana que realizaban esta práctica hervían los frijoles de una a dos veces al día, mientras que en la zona rural, la mayoría de estas familias hervían los frijoles dos veces al día (Cuadro 3).
Las familias de ambas zonas en estudio que realizaban la práctica de congelar los frijoles refirieron dos formas de congelación: dividir en porciones con la cantidad que ocupan para un día en bolsas o recipientes (19 en la zona rural y 13 en la zona urbana) y congelar la totalidad de los frijoles sobrantes el día de la preparación (5 en la zona rural y 12 en la zona urbana).

Con respecto a la forma de descongelación de los frijoles se puede identificar una diferencia en cuando a la práctica seguida en las poblaciones estudiadas (Cuadro 4). El método de descongelación de los frijoles en refrigeración fue el más utilizado en la zona rural y la práctica de descongelarlos en el microondas era más utilizada en la zona urbana, mientras que la descongelación a temperatura ambiente o como parte del método de calentamiento era similar en las poblaciones estudiadas.

La cantidad de frijoles que recalentaban las familias que acostumbran a conservarlos en el congelador difiere por zona, pues en la zona rural la frecuencia de familias que calentaban la cantidad de

Cuadro 3. Frecuencia diaria de hervido de frijoles según condición geográfica. Jesús de Atenas y La Unión de Tres Ríos, Costa Rica. 2005.

\begin{tabular}{lcccc}
\hline \multirow{2}{*}{ Número de veces } & \multicolumn{2}{c}{ Rural } & \multicolumn{2}{c}{ Urbano } \\
\cline { 2 - 5 } & Absoluto (n) & Relativo (\%) & Absoluto (n) & Relativo (\%) \\
\hline Una vez al día & 15 & 27 & 33 & 45 \\
Dos veces al día & 38 & 68 & 35 & 48 \\
Tres veces al día & 3 & 5 & 5 & 7 \\
Total & 56 & 100 & 73 & 100 \\
\hline
\end{tabular}

Cuadro 4. Forma de descongelación de frijoles según condición geográfica. Jesús de Atenas y La Unión de Tres Ríos, Costa Rica. 2005.

\begin{tabular}{lcccccc}
\hline \multirow{2}{*}{$\begin{array}{l}\text { Método de } \\
\text { descongelación }\end{array}$} & \multicolumn{4}{c}{ Frecuencia } & \multicolumn{2}{c}{ Total } \\
\cline { 2 - 7 } & \multicolumn{2}{c}{ Rural } & \multicolumn{2}{c}{ Urbano } \\
\cline { 2 - 7 } & Absoluto (n) & Relativo (\%) & Absoluto (n) & Relativo (\%) & Absoluto (n) & Relativo (\%) \\
\hline Refrigeración & 12 & 50 & 8 & 32 & 20 & 42 \\
Temperatura ambiente & 6 & 25 & 4 & 16 & 10 & 20 \\
Microondas & 2 & 8 & 9 & 36 & 11 & 22 \\
Método de calentamiento & 4 & 17 & 4 & 16 & 8 & 16 \\
Total & 24 & 100 & 25 & 100 & 49 & 100 \\
\hline
\end{tabular}


frijoles consumida en un día (12/24), era igual a las familias que calentaban la cantidad que requerían para un tiempo de comida (12/24), mientras que en la zona urbana se acostumbraba a calentar la cantidad consumida por tiempo de comida (20/25).

El uso de la refrigeración para conservar los frijoles en este estudio se explica debido a que las familias preparaban los frijoles para un promedio de 5,5 días, tiempo que permanecen sin descomponerse utilizando métodos adecuados y prácticos de manipulación. Por su parte, la congelación permite mantener los frijoles en buenas condiciones por periodos largos de tiempo, en este caso en particular las personas conservaban los frijoles por al menos seis días; sin embargo, pese a que es una práctica recomendada (Murillo y Rodríguez, 2004) su baja utilización como técnica de conservación radica en el manejo poco práctico que se le puede dar al alimento congelado, pues para ello requiere de una planificación previa.

La conservación en el refrigerador y el congelador son prácticas adecuadas, ya que permite mantener los frijoles en buenas condiciones y la retención de los nutrientes es muy alta (Martins y Silva, 2003; Lean y Fox, 206). Sin embargo, un proceso de descongelamiento y recalentamiento inadecuado puede ocasionar algunas pérdidas en el contenido de nutrientes; por ello, para conservar la mayoría de los nutrientes en los frijoles se recomienda descongelar los frijoles en el refrigerador y realizar un único recalentamiento de la cantidad que van a consumir en el tiempo de comida.

Por el contrario, conservar los frijoles utilizando el hervido diario va en detrimento de la calidad nutricional de los frijoles (Lean y Fox, 2006), ya que están expuestos al calor por más tiempo, al menos dos veces al día, por el periodo de duración de los mismos. Pese a ello, las personas realizaban esa práctica por costumbre y porque desconocían los perjuicios en relación con la calidad nutricional.

Según Campos et al. (1999), la congelación es el método más adecuado parar preservar los frijoles, ya que aumenta la vida útil de estos y permite su disponibilidad permanente, lo cual es importante en el caso de familias pequeñas como las que se presentaron en este estudio (promedio de cuatro miembros tanto en el área rural como en el área urbana) .

Las prácticas de conservación también fueron clasificadas en relación con la retención de nutrientes y la mayoría realizaba prácticas recomendadas, lo cual es importante porque de esta forma se prolonga la vida útil y se consume un grano con una mayor calidad nutricional.

De las familias estudiadas 287 realizaban una conservación recomendada. De estas un $64 \%$ eran familias de la zona rural y un $57 \%$ eran familias del área urbana.

Se considera que los resultados de esta investigación proporcionan información basal para realizar estudios posteriores que permitan identificar el efecto que tienen las prácticas de preparación y conservación sobre el valor nutritivo de los frijoles y para establecer recomendaciones adecuadas sobre el uso de los mismos; de forma que se incremente el aporte de nutrientes de este alimento a las dietas de la población costarricense.

\section{LITERATURA CITADA}

Andlauer, A., C. Wilfried, V. Stehle, P. Peter, G. Fürst, y D. Peter. 1998. Chemoprevention - a novel approach in diabetics. Clin. Nut. Met. Care 16:539-547.

Badui, S. 2006. Química de alimentos. $4^{\text {a }}$ ed. Pearson Educación. México, D.F.

Bazzano, L.A., J. He, L.G. Ogden, C. Loria, S. Vupputuri, L. Myers, y P. Whelton. 2001. Legume consumption and risk of coronary Herat disease in US men and women. Arch. Int. Med. 161:2573-2578.

Blanco, A., y R. Bressani. 1991. Biodisponibilidad de aminoácidos en el frijol. Arch. Lat. Nutr. 41:38-52.

Bressani, R. 1989. Revisión sobre la calidad del grano de frijol. Arch. Lat. Nutr. 39:419-438.

Bresanni, R., y L. Elías. 1988. Valor nutritivo de las leguminosas para los humanos y los animales. Publicación INCAP E-1344, Guatemala.

Bressani, R., E. Hernández, y J. Branham. 1988. Relationship between content and intake of bean poliphenolics and protein digestibility in humans. Plant Foods Hum. Nutr. 38:5-21.

Campos, E., A. Fernández, I. Sánchez, T. Sancho, y C. Villalobos. 1999. Diagnóstico para el diseño de una campaña educativa tendiente a incrementar el consumo de frijoles en una comunidad urbana de clase media de Costa Rica. Seminario de Lic, Universidad de Costa Rica, San José, Costa Rica.

Carbonaro, M., G. Grant, M. Cappelloni, y A. Pusztai. 2000. Pespectives into factors limiting in vivo digestion of legume proteins: antinutritional compounds or storage protein?. J. Agric. Food Chem. 48:742-749. 
Chau, C., y C. Cheung. 1997. Effects of various processing methods on antinutrients in vitro digestibility of protein starch of two chinese indigenous. J. Agric. Food Chem. 45:4773-4776.

Dang, J., J. Arcot, y A. Shrestha. 2000. Folate retention in selected processed legumes. Food Chem. 68:295-298.

Deshpande, S.S. y M. Cheryan. 1983. Changes in phitic acid, tannins and trypsin inhibitory activity on soaking of dry beans (Phaseolus vulgaris L.). Nutr. Rep. Int. 27 (2):371-377.

Dumani, M., y S. Rodríguez. 2000. De todo con frijoles. Escuela de Nutrición, Universidad de Costa Rica, San José, Costa Rica.

Elías, L. s.f. Valor nutritivo del frijol (Phaseolus vulgaris) su potencial y limitaciones. Instituto de Nutrición de Centroamérica y Panamá (INCAP), Guatemala.

Fernández, A.C., W. Nishida, y R.P. Da Costa Proenca. 2010. Influence of soaking on the nutrition quality of common bean (Phaseolus vulgaris) cooked with or whitout the soaking water: a review. Int. J. Food Sci. Tec. 45:2209-2218.

FAO (Food and Agriculture Organization). 1982. Las leguminosas en la nutrición humana. Organización de Las Naciones Unidas para la Agricultura y la Alimentación, Roma, Italia.

FAO (Food and Agriculture Organization). 1990. Utilización de alimentos tropicales: Frijoles tropicales. (47/4). Organización de Las Naciones Unidas para la Agricultura y la Alimentación, Roma, Italia.

García, R.W.D. 1997. Práticas e comportamento alimentar no meio urbano: um estudo no centro da cidade de São Paulo. Cadernos de Saúde Pública 13(3):455-467.

García, R.W.D. 2003. Reflexos da glo. Cadernos balização na cultura alimentar: considerações sobre as mudanças na alimentação urbana. Rev. Nutr. 16(4):483-492.

Guzmán-Maldonado, S.H., J.A. Acosta-Gallegos, M.A. Alvarez-Muñoz, S. García-Delgado, y G. Loarca-Piña. 2002. Calidad alimentaria y potencial nutracético del frijol (Phaseolus vulgaris L.). Agric. Téc. Méx. 28:159-173.

Guzmán, M., H. Salvador, J. Zaragoza, y M. Juárez. 1996. Remojo de dos sales y tiempo de cocción de tres variedades de frijol. Agrociencia 30:201-205.

Hoppner, K., y B. Lamp. 1993. Folate retention in dried legumes after diferent methods of meal preparation. Food Res. Int. 26:45-48.

Jiménez, L., y V. Leal. 1996. Efecto del remojo del frijol negro (Phaseolus vulgaris) sobre la cantidad de taninos y la biodisponibilidad in vitro de hierro. Proyecto de Lic, Universidad de Costa Rica, San José, Costa Rica.

Kolonel, L., J. Hankin, A. Whittemore, A. Wu, R. Gallagher, y L. Wilkens. 2000. Vegetales, fruits, legumes and prostate cancer: a multiethnic casecontrol study. Can Epid Bio Prev. 9:795-804.

Kon, S. 1979. Effect of soaking temperature on cooking and nutritional quality of beans. J. Food Sci. 44:1329-1334.

Lean, M.E.J., y B.R. Fox. 2006. Fox and Cameron's food science, nutrition and health. $7^{\circ}$ ed. CRC Press, USA.

León, L., L. Elías, y R. Bressani. 1992. Effect of salt solutions on the cooking time, nutritional and sensory characteristics of common beans (Phaseolus vulgaris). Food Res. Int. 26:131-136.

López, H.W., F. Leenhardt, Ch. Coudray, y C. Remesy. 2002. Minerals and phytic acid interactions: is it a real problem for human nutrition?. Int. J. Food Sci. Tec. 37:727-739.

Martins, R., y C. Silva. 2003. Kinetics of frozen stored green bean (Phaseolus vulgaris L) quality changes: texture, vitamin $C$, reducing sugars, and starch. J. Food. Sci. 68:2232-2237.

Menchú, M.T., y H. Méndez. 2006. Tabla de composición de alimentos de Centroamérica. $2^{\mathrm{a}}$ ed. INCAP/ OPS, Guatemala.

Messina, M. 1999. Legumes and soybeans: Overview of their nutritional prolifes and health effects. Am. J. Clin. Nut. (Suppl.) 70:439S-50S.

Ministerio de Salud. 2001. Encuesta Nacional de Consumo de Alimentos. Ministerio de Salud, San José, Costa Rica.

Murillo, A., y S. Rodríguez. 2004. Frijoles: prácticas factibles para madres de escolares. Agron. Mesoam. 15:301-313.

Osorio, P., G. Méndez, E. Agama, J. Islas, J. Sánchez, y K. Bello. 2003 Biodisponibilidad del almidón en dos variedades comerciales de frijol (Phaseolus vulgaris) L.) y en frijoles industrializados. Agrociencia 37: 565-573.

Payne, J., V. Harger, G. Shugart, y M. Theis. 1994. West's and wood's introduction to foodservice. $7^{\circ}$ ed. Maxwell Macmillan International, NY, USA.

Prodanov, M., y I. Sierra. 2004. Influence of soaking and cooking on the thiamin, riboflavin and niacin contents of legumes. Food Chem. 48:271-278.

Ramírez-Cárdenas, L., y N.M. Costa. 2008. Efeito do processamento doméstico sobre o teor de nutrientes e de fatores antinutricionais de diferentes cultivares de feijão comum. Ciênc. Tecnol. Aliment. 28:200-213. 
Ramírez, J.C., A.J. Leonel, y I.E. Rangel. 2011. El frijol (Phaseolus vulgaris): su importancia nutricional y como fuente de fitoquímicos. Revista Fuente 3(8):5-9.

Reynoso, R., M.C. Ríos, I. Torres, J.A. Acosta, A.C. Palomino, M. Ramos, E. González, y S.H. Guzmán. 2007. El consumo de frijol común (Phaseolus vulgaris) y su efecto sobre el cáncer de colon en ratas Sprague-Dawley. Agric. Téc. Méx. 33:43-52.

Rodríguez, L., y X.E. Fernández-Rojas. 2003. Los frijoles (Phaseolus vulgaris): Su aporte a la dieta del costarricense. Acta Médica Costarricense 45:120-125.

Ryan, E., K. Galvin, T.P. O'Connor, A.R. Maguire, y N.M. O'Brien. 2007. Phytosterol, squalene, tocopherol content and fatty acid profile of selected seeds, grains, and legumes. Plant Foods Hum. Nutr. 62:85-91.

Serrano, J., y I. Goñi. 2004. Papel del frijol negro Phaseolus vulgaris en el estado nutricional de la población guatemalteca. Arch. Lat. Nutr. 54:1-17.
Slavin, J. 2000. Mechanisms for the impact of whole grain foods on cancer risk. J. Amer. Coll. Nutr. 19(3 suppl):300S-307S.

Singh, P.N., y G.E. Fraser. 1998. Dietary risk factors for colon cancer in a low-risk population. Amer. J. Epid. 148:761-774.

Tacsan, Y. 2001. Efecto del remojo de frijoles (Phaseolus vulgaris) sobre la concentración de fitatos y la disponibilidad in vitro del hierro. Proyecto Lic, Universidad de Costa Rica, San José, Costa Rica.

Ulloa, J.A., P.R. Ulloa, J.C. Ramírez, y B.E. Ulloa. 2011. El frijol (Phaseolus vulgaris): su importancia nutricional y como fuente de fitoquímicos. Revista Fuente 3(8): $5-9$.

USDA (United States Department of Agriculture). 2014. Tabla de composición de alimentos. Departamento de Agricultura de los Estados Unidos. http://www.nal. usda.gov. (Consultado 15 set. 2014). 